\title{
Translation and Linguistic Validation of the Korean Version of the Treatment Satisfaction Visual Analogue Scale and the Overactive Bladder Satisfaction With Treatment Questionnaire
}

\author{
Ha Na Lee ${ }^{1}$, Ji Yun Chae 2 , Hyo Serk Lee ${ }^{3}$, Min Soo Choo ${ }^{4}$, Min Gu Park ${ }^{5}$, Seo Yeon Lee ${ }^{6}$, Seung-June $\mathrm{Oh}^{7}$, Sung Yong Cho ${ }^{8}$ \\ ${ }^{1}$ Department of Urology, Seoul Metropolitan Seonam Hospital, Seoul, Korea \\ ${ }^{2}$ Mizlove Female Urology Clinic, Seoul, Korea \\ ${ }^{3}$ Department of Urology, Cheil General Hospital \& Women's Healthcare Center, Kwandong University College of Medicine, Seoul, Korea \\ ${ }^{4}$ Department of Urology, Hallym University Dongtan Sacred Heart Hospital, Hwaseong, Korea \\ ${ }^{5}$ Department of Urology, Inje University Seoul Paik Hospital, Seoul, Korea \\ ${ }^{6}$ Department of Urology, Myongii Hospital, Seonam University College of Medicine, Goyang, Korea \\ ${ }^{7}$ Department of Urology, Seoul National University Hospital, Seoul, Korea \\ ${ }^{8}$ Department of Urology, Seoul Metropolitan Government - Seoul National University Boramae Medical Center, Seoul, Korea
}

Purpose: This study reports the development of the Korean Version of the Treatment Satisfaction Visual Analogue Scale (TSVAS) and the Overactive Bladder Satisfaction with Treatment Questionnaire (OAB-SAT-q) based on the original versions, with subsequent linguistic validation by Korean patients with overactive bladder receiving active treatment from a physician. Methods: Translation and linguistic validation were performed in 2016. The validation process included permission for translation, forward translation, reconciliation, backward translation, cognitive debriefing, and proofreading. The original versions of the TS-VAS and OAB-SAT-q were independently translated into Korean by 2 bilingual translators and were then reconciled into a single version. The third bilingual translator performed a backward translation of the reconciled version into English. A trained interviewer and 5 Korean-speaking patients with OAB carried out the cognitive debriefing.

Results: During the forward translation process, the terms used in the 2 questionnaires were adjusted to use more appropriate expressions in the Korean language than were used in the original versions. During the backward translation process, no changes were made in terms of semantic equivalence. In the cognitive debriefing session, 5 patients were asked to fill in the answers within 8 minutes; most of them reported that the translated questions were clear and easy to understand.

Conclusions: The present study presents successful linguistic validation of the Korean version of the TS-VAS and OAB-SATq, which could be useful tools for evaluating treatment satisfaction in patients.

Keywords: Linguistics; Patient Satisfaction; Visual Analogue Scale; Overactive Bladder, Overactive

- Research Ethics: This study was approved by the Institutional Review Board of the Seoul Metropolitan Government - Seoul National University Boramae Medical Center (approval number: 26-2016-60).

- Conflict of Interest: MSC and SYC, members of the Editorial Board of INJ, are the fourth and corresponding authors of this article. However, they played no role whatsoever in the editorial evaluation of this article or the decision to publish it. No potential conflict of interest relevant to this article was reported.

Corresponding author: Sung Yong Cho (iD https://orcid.org/0000-0001-9271-6951 Department of Urology, Seoul Metropolitan Government-Seoul National University Boramae Medical Center, 20 Boramae-ro 5-gil, Dongjak-gu, Seoul 07061, Korea

E-mail: kmoretry@daum.net / Tel: +82-2-870-2394 / Fax: +82-2-870-3863 Submitted: September 14, 2017 / Accepted after revision: December 1, 2017 


\section{INTRODUCTION}

Patient-reported outcomes (PROs) are important for assessing patients' satisfaction with the treatment they receive in clinics. PROs can help guide treatment with regard to the risks and benefits of an intervention in the course of treatment [1-3]. Therefore, it is important to develop a questionnaire that accurately represents PROs in order to facilitate decision-making about treatment. The Treatment Satisfaction Visual Analogue Scale (TS-VAS) and Overactive Bladder Satisfaction with Treatment Questionnaire (OAB-SAT-q) have been used for evaluating patients' satisfaction with their treatment. The visual analogue scale (VAS) is a widely accepted method for measuring the effects of disease and medical interventions with special reference to quality of life (QoL) [4-6]. The OAB-SAT-q was developed based on a review of existing instruments for measuring treatment satisfaction, input from physicians experienced in treating overactive bladder (OAB), and patient feedback.

To the best of our knowledge, no Korean versions of the TSVAS and OAB-SAT-q were previously available. Therefore, we developed a Korean version based on the original versions, with subsequent linguistic validation in Korean patients with $\mathrm{OAB}$ who were receiving active treatment from a physician.

\section{MATERIALS AND METHODS}

\section{Original TS-VAS and OAB-SAT-q}

The VAS was described for the first time in 1921 by Hayes and Patterson [7]. The VAS is a simple and frequently used method to evaluate variations in pain intensity [8]. The VAS for satisfaction is a simple and valid instrument to quantify a patient's satisfaction after a treatment [9], and consists of a 100-mm-long horizontal line, with 2 descriptors representing extremes of satisfaction at the beginning and at the end of the line. Patients rate their satisfaction by making a vertical mark on the 100$\mathrm{mm}$ line. The measurement in millimeters is converted to the same number of points, ranging from 0 to 100 points.

The OAB-SAT-q was developed as a way to evaluate the satisfaction of patients with $\mathrm{OAB}$ as part of the modular International Consultation on Incontinence Questionnaire [10]. The OAB-SAT-q is a disease-specific 11-item questionnaire designed to assess patients' satisfaction with their treatment in the clinical setting. The OAB-SAT-q items include effectiveness, convenience, side effects, preference for treatment, willingness to undergo the treatment again, and an assessment of global satisfaction. The response options vary depending on the content of the question and are presented on 4-, 5-, and 6-point Likert scales with anchors, such as "extremely dissatisfied" to "extremely satisfied" and "extremely bothersome" to "not at all bothersome" [10].

\section{Translation and Linguistic Validation}

This study was approved by the Institutional Review Board of the Seoul Metropolitan Government - Seoul National University Boramae Medical Center (approval number: 26-2016-60). The process was designed to obtain a translated Korean version of the questionnaire that would be conceptually equivalent to the original. The translation was appropriate in terms of cultural adaptation and was supervised by the project leader of the panel. The panel consisted of 7 Korean experts who performed a typical linguistic validation process.

\section{Permission}

Before the study commenced, the panel contacted the copyright holder and acquired permission to translate and use the TSVAS and OAB-SAT-q after conducting the linguistic validation process.

\section{Forward Translation}

The original versions of the TS-VAS and OAB-SAT-q were translated into the Korean language (versions 1.0a and 1.0b) by 2 bilingual translators who did not have medical licenses. The translations were performed independently, and the translators were not permitted to contact each other during the translation process.

\section{Reconciliation by the Panel}

The independently translated versions in the Korean language (versions $1.0 \mathrm{a}$ and $1.0 \mathrm{~b}$ ) were reconciled into a single version after several panel meetings (version 1.1). The panel discussed comprehension difficulties due to subtle differences in the nuances of the selected words and phrases.

\section{Backward Translation}

The reconciled version of the TS-VAS and OAB-SAT-q in the Korean language (version 1.1) was sent to a third bilingual translator. This translator translated it backward into English, and the results were compared to the original English versions of the TSVAS and OAB-SAT-q. After the third translator completed the backward translation, the panel discussed the discrepancies be- 
tween the original and the backward-translated versions of the TS-VAS and OAB-SAT-q. Some changes were made in the reconciled TS-VAS and OAB-SAT-q in the Korean language based on the outcomes of several discussions (version 1.2).

\section{Cognitive Debriefing}

Version 1.2 of the questionnaires was evaluated by 5 Koreanspeaking patients with $\mathrm{OAB}$, who were asked whether there were any clarity issues, culturally inappropriate expressions, or difficulties in understanding the questions. The panel chose a trained interviewer who conducted a standardized in-depth interview of a small number of patients. The debriefing interviews involved paraphrasing each question of the questionnaire and indicating whether the participants had any trouble understanding the question or felt that any terms were confusing. Subsequently, the panel discussed the feedback from the 5 patients with $\mathrm{OAB}$ and agreed upon a new version based on the issues raised (version 1.3).

\section{Proofreading}

Version 1.3 was proofread to check spelling, grammar, and formatting. This resulted in the final version of the TS-VAS and OAB-SAT-q in the Korean language (version 1.4).

\section{RESULTS}

\section{Forward Translation and Reconciliation}

As shown in Table 1, the word "satisfied" in the title of the original version of the TS-VAS was translated as "manjok" ('satisfaction') by the 2 translators. As shown in Table 2, the 11 questions of the OAB-SAT-q were translated into Korean. Question 1,
"How satisfied or dissatisfied are you with the way the treatment relieves your symptoms?" in the original version was directly translated as "jeungsangeul wanhwasikineundeisseoseo manjok hogeun bulmanjok hasimnikka?" The phrase "How satisfied or dissatisfied are you with the treatment?" was repeated in questions 1-3. Question 2, "How satisfied or dissatisfied are you with the amount of time it takes the treatment to start working?" in the original version was translated as "chiryohyogwaga natananeundekkaji geollineun sigane manjok hogeun bulmanjok hasimnikka?" Question 3, "How satisfied or dissatisfied are you with the effectiveness of the treatment, compared to what you expected?" in the original version, was translated as "gidaehaetdeon dae bihayeo chiryoui hyogwae eoneujeongdo manjok hogeun bulmanjok hasimnikka?" The phrase "compared to what you expected" was translated as "gidaehaetdeon geonmankeumebihae" ('compared to your expectation') in translation 1, and as "yesanghaetdeon geotboda" ('than you expected') in translation 2. The panel reconciled it as "gidaehaetdeon dae bihayeo" ('expected compared to'). Question 4, "How convenient or inconvenient is it to take the treatment as instructed?" in the original version, was directly translated as "eoneujeongdo pyeolli hogeun bulpyeonhasyeotseumnikka?" The word "convenient" was translated as "yongi" ('easy') and "pyeonhada" ('comfortable') in translations 1 and 2, respectively. The panel reconciled it as "pyeolli" ('convenient'). The word "extremely" was finally translated as "jigeuki" rather than "wanjeon" ('complete') and "aju” ('very'). Question 5, "How often did you experience side effects from your treatment?" in the original version, was directly translated as "eolmana jaju chiryo bujagyongeul gyeongheom hasyeotseumnikka?” This question was translated in nearly the same way by both translators. Question 6, "How bother-

Table 1. Reconciliation after 2 forward translations of Treatment Satisfaction Visual Analogue Scale

\begin{tabular}{|c|c|c|c|}
\hline US English item & $\begin{array}{l}\text { First forward Korean } \\
\text { translation }\end{array}$ & $\begin{array}{l}\text { Second forward Korean } \\
\text { translation }\end{array}$ & $\begin{array}{l}\text { Reconciled forward Korean } \\
\text { translation }\end{array}$ \\
\hline $\begin{array}{l}\text { EXAMPLE OF TREATMENT } \\
\text { SATISFACTION VISUAL } \\
\text { ANALOGUE SCALE }\end{array}$ & 치료 만족 시각상사척도 예시 & 예) 치료 만족도 시각 상사 척도 & \\
\hline $\begin{array}{l}\text { Please place a vertical mark on the } \\
\text { line to indicate your answer to the } \\
\text { question below }\end{array}$ & $\begin{array}{l}\text { 다음과 같은 질문을 답하기 위해 } \\
\text { 해당 선에 수직선을 표기하십시오. }\end{array}$ & $\begin{array}{l}\text { 선 위에 세로선을 표시하여 다음 } \\
\text { 질문에 답변하시기 바랍니다. }\end{array}$ & $\begin{array}{l}\text { 다음 질문을 답하기 위해 선 위에 } \\
\text { 세로선을 표기해주시기 바랍니 } \\
\text { 다. }\end{array}$ \\
\hline $\begin{array}{l}\text { Are you satisfied with your } \\
\text { treatment? }\end{array}$ & 귀하의 치료에 만족하십니까? & 귀하께서는 치료에 만족하십니까? & 귀하는 치료에 만족하십니까? \\
\hline No, not at all & 전혀 그렇지 않음 & 아니요, 절대 만족하지 않습니다. & 아니요, 전혀 그렇지 않음 \\
\hline Yes, completely & 완전히 만족함 & 네, 전적으로 만족합니다. & 네, 전적으로 만족함 \\
\hline
\end{tabular}


Table 2. Reconciliation after 2 forward translations of Overactive Bladder Satisfaction with Treatment Questionnaire (OAB-SAT-q)

\begin{tabular}{|c|c|c|c|}
\hline US English item & $\begin{array}{c}\text { First forward Korean } \\
\text { translation (Translation 1) }\end{array}$ & $\begin{array}{l}\text { Second forward Korean } \\
\text { translation (Translation 2) }\end{array}$ & $\begin{array}{l}\text { Reconciled forward } \\
\text { Korean translation }\end{array}$ \\
\hline OAB-SAT-q & $\begin{array}{l}\text { 과민성방광 치료 만족도 설문 } \\
\text { 조사 확인(인증) }\end{array}$ & & $\begin{array}{l}\text { 과민성방광 치료만족도 설 } \\
\text { 문지 }\end{array}$ \\
\hline Instructions & 설명 & 설명서 & 지시사항 \\
\hline $\begin{array}{l}\text { Please think about how satisfied you have been } \\
\text { with the treatment you have received in the study. }\end{array}$ & $\begin{array}{l}\text { 귀하가 실험에 참여하며 받은 } \\
\text { 치료에 대해 얼마나 만족했 } \\
\text { 는지를 생각해보십시오. }\end{array}$ & $\begin{array}{l}\text { 귀하께서는 이 연구에서 받으 } \\
\text { 신 치료에 어느 정도 만족하 } \\
\text { 시는지 생각해 보시기 바랍 } \\
\text { 니다. }\end{array}$ & $\begin{array}{l}\text { 귀하는 이번 연구에서 받은 } \\
\text { 치료에 어느 정도 만족하 } \\
\text { 는지 생각해 보시기 바랍 } \\
\text { 니다. }\end{array}$ \\
\hline $\begin{array}{l}\text { The questions below will ask you to rate how satisfied } \\
\text { or dissatisfied you are with your treatment for } \\
\text { overactive bladder. }\end{array}$ & $\begin{array}{l}\text { 아래의 질문들은 귀하의 과민 } \\
\text { 성방광 치료에 대한 만족(또 } \\
\text { 는 불만족) 정도를 평가하기 } \\
\text { 위한 항목들입니다. }\end{array}$ & $\begin{array}{l}\text { 하기 질문들에 귀하의 과민성 } \\
\text { 방광 치료 만족도를 평가하 } \\
\text { 여 주시기 바랍니다. }\end{array}$ & $\begin{array}{l}\text { 아래의 질문들은 귀하가 과 } \\
\text { 민성방광 치료에 어느 정 } \\
\text { 도 만족 또는 불만족 하는 } \\
\text { 지를 평가할 것입니다. }\end{array}$ \\
\hline
\end{tabular}

Thinking about the past four weeks, check one box for 지난 4주 동안 귀하의 치료 경 귀하께서는 지난 4 주를 생각 지난 4 주 동안, 귀하의 치료 each item that best reflects your treatment experience.

\section{험을 가장 정확하게 반영하}

는 칸을 항목별로 표시하여 주십시오.

하시어 귀하의 치료 경험을

가장 잘 묘사하는 항목을 선 택하여 주시기 바랍니다.

경험을 가장 정확하게 반 영하는 칸을 항목별로 표 시하여 주십시오.

1. 귀하가 받는 치료의 증상 1 . 귀하께서는 치료가 증상 완 1 . 치료가 귀하의 증상을 완 완화 효과에 얼마나 만족 화하는데 있어서 어느 정도 화시키는데 있어서 어느 혹은 불만족하십니까? 만족 혹은 불만족 하신가 요?

정도 만족 혹은 불만족하 십니까?

2. 치료가 작용하기까지 걸리 2. 귀하께서는 치료하시는데 2. 치료 효과가 나타나는 데 는 시간에 얼마나 만족 혹 기여되는 시간에 있어서 어 까지 걸리는 시간에 어느 은 불만족하십니까? 느 정도 만족 혹은 불만족 정도 만족 혹은 불만족 하신가요? 하십니까?

3. How satisfied or dissatisfied are you with the effectiveness of the treatment, compared to what you expected?

Extremely dissatisfied

Very dissatisfied

Dissatisfied

Satisfied

Very satisfied

Extremely satisfied

4. How convenient or inconvenient is it to take the treatment as instructed?

Extremely inconvenient

Very inconvenient

Inconvenient

Convenient

Very convenient

Extremely convenient

5. How often did you experience side effects from your treatment?

Never

A little of the time
3. 귀하가 기대했던 것만큼에 3. 귀하께서는 치료 효과에 대 3. 귀하가 기대했던 바에 비 비해 치료의 효과에 얼마나 해서 예상했던 것 보다 어 하여 치료 효과에 어느 만족 혹은 불만족하십니까? 느 정도 만족 혹은 불만족 정도 만족 혹은 불만족하 하신가요? 십니까?

$\begin{array}{lll}\text { 완전 불만족 } & \text { 아주 매우 불만족스럽다 } & \text { 지극히 불만족 } \\ \text { 매우 만족 } & \text { 아주 불만족스럽다 } & \text { 매우 불만족 } \\ \text { 불만족 } & \text { 불만족스럽다 } & \text { 불만족 } \\ \text { 만족 } & \text { 만족스럽다 } & \text { 만족 } \\ \text { 매우 만족 } & \text { 아주 만족스럽다 } & \text { 매우 만족 } \\ \text { 완전 만족 } & \text { 아주 매우 만족스럽다 } & \text { 지극히 만족 }\end{array}$

4. 설명지에 명시된 대로 치료 4. 설명서 대로 치료를 받으시 4. 귀하가 치료를 받는데 어 를 받는 것이 얼마나 용이 는 것이 어느 정도 편하시 느 정도 편리 혹은 불편 혹은 불편하십니까? 거나 불편하신가요? 하셨습니까?

완전 불편함

아주 매우 불편하다

지극히 불편함

매우 불편함

매우 불편함

불편함

불편하다

불편함

\section{용이함}

편하다

매우 용이함

아주 편하다

편리함

완전 용이함

아주 매우 편하다

매우 편리함

지극히 편리함

5. 귀하는 얼마나 자주 치료에 5. 귀하께서는 얼마나 자주 치 5. 귀하는 얼마나 자주 치료 의한 부작용을 경험하셨습 료 부작용을 경험하시나요? 부작용을 경험하셨습니 니까?

까?

부작용 경험 없음

한번도 없다

전혀 없음

매우 가끔

거의 없다
아주 불편하다 
Table 2. Continued

\begin{tabular}{|c|c|c|c|}
\hline US English item & $\begin{array}{c}\text { First forward Korean } \\
\text { translation (Translation 1) }\end{array}$ & $\begin{array}{l}\text { Second forward Korean } \\
\text { translation (Translation 2) }\end{array}$ & $\begin{array}{l}\text { Reconciled forward } \\
\text { Korean translation }\end{array}$ \\
\hline Some of the time & 가끔 & 가끔 있다 & 가끔 있음 \\
\hline A good bit of time & 자주 & 그런 적이 여러 번 있다 & 상당히 있음 \\
\hline Most of time & 매우 자주 경험함 & 거의 있다 & 매우 자주 있음 \\
\hline All of the time & 항상 & 항상 있다 & 항상 있음 \\
\hline $\begin{array}{l}\text { 6. How bothersome are the side effects of the } \\
\text { treatment you take to treat your overactive bladder? }\end{array}$ & $\begin{array}{l}\text { 6. 귀하의 과민성방광 치료에 } \\
\text { 의한 부작용이 얼마나 불편 } \\
\text { 하십니까? }\end{array}$ & $\begin{array}{l}\text { 6. 과민성방광을 치료하는데 } 6 \\
\text { 있어서 부작용이 귀하께 어 } \\
\text { 느 정도의 불편함을 주나 } \\
\text { 요? }\end{array}$ & $\begin{array}{l}\text { 귀하는 과민성방광 치료 } \\
\text { 를 받는데 있어서 (치료) } \\
\text { 부작용이 얼마나 불편하 } \\
\text { 십니까? }\end{array}$ \\
\hline Extremely bothersome & 완전히 불편함 & 아주 매우 불편하다 & 지극히 불편함 \\
\hline Very bothersome & 매우 불편함 & 많이 불편하다 & 매우 불편함 \\
\hline Somewhat bothersome & 적당히 불편함 & 어느 정도 불편하다 & 다소 불편함 \\
\hline A little bothersome & 약간 불편함 & 조금 불편하다 & 약간 불편함 \\
\hline Not at all bothersome & 전혀 불편하지 않음 & 전혀 불편하지 않다 & 전혀 불편하지 않음 \\
\hline $\begin{array}{l}\text { 7. To what degree have side effects affected your } \\
\text { overall satisfaction with the treatment? }\end{array}$ & $\begin{array}{l}\text { 7. 부작용이 귀하의 전반적인 } \\
\text { 치료 만족도에 얼마나 영향 } \\
\text { 을 미쳤습니까? }\end{array}$ & $\begin{array}{l}\text { 7. 부작용이 귀하의 전체적인 } 7 \\
\text { 치료법 만족도에 어느 정도 } \\
\text { 의 영향을 주었나요? }\end{array}$ & $\begin{array}{l}\text { 귀하의 전반적인 치료 만 } \\
\text { 족도에 부작용이 얼마나 } \\
\text { 영향을 미쳤습니까? }\end{array}$ \\
\hline A great deal & 매우 많이 & 상당히 많이 주었습니다 & 상당히 많이 \\
\hline Quite a bit & 상당히/적당히 & 상당히 주었습니다 & 꽤 많이 \\
\hline Somewhat & 약간 적게 & 어느 정도 주었습니다 & 다소 \\
\hline Minimally & 매우 적게 & 최소 주었습니다 & 극히 일부분 \\
\hline Not at all & 전혀 영향 없음 & 전혀 주지 않았습니다 & 전혀 영향 없음 \\
\hline $\begin{array}{l}\text { 8. Do you prefer the treatment that you received since } \\
\text { entering this study to the treatment you received } \\
\text { before the study? }\end{array}$ & $\begin{array}{l}\text { 8. 귀하가 실험 참가 후에 받 } \\
\text { 으신 치료를 실험 참가 전 } \\
\text { 에 받으신 치료보다 선호하 } \\
\text { 십니까? }\end{array}$ & $\begin{array}{l}\text { 8. 귀하께서는 이번 연구에서 } 8 \\
\text { 받으신 치료법을 이전에 받 } \\
\text { 으셨던 치료법보다 더 선호 } \\
\text { 하십니까? }\end{array}$ & $\begin{array}{l}\text { 귀하는 이전에 받았던 치 } \\
\text { 료방법보다 이번 연구에 } \\
\text { 서 받은 치료방법을 더 } \\
\text { 선호 하십니까? }\end{array}$ \\
\hline Never been treated before for overactive bladder & $\begin{array}{l}\text { 과민성방광 치료를 받아본 } \\
\text { 적이 없음 }\end{array}$ & $\begin{array}{l}\text { 이번 연구 이전에 과민성방 } \\
\text { 광 치료를 받은 이력이 } \\
\text { 전혀 없습니다 }\end{array}$ & $\begin{array}{l}\text { 과민성방광 치료를 받아 } \\
\text { 본 적이 없음 }\end{array}$ \\
\hline Definitely prefer my previous treatment & $\begin{array}{l}\text { 실험 참가 전에 받은 치료 } \\
\text { 를 확실하게 선호함 }\end{array}$ & $\begin{array}{l}\text { 분명히 이번 연구 이전에 받 } \\
\text { 은 치료법을 선호합니다 }\end{array}$ & $\begin{array}{l}\text { 이전에 받은 치료를 분명 } \\
\text { 히 선호함 }\end{array}$ \\
\hline Slight preference for my previous treatment & $\begin{array}{l}\text { 실험 참가 전에 받은 치료 } \\
\text { 를 약간 선호함 }\end{array}$ & $\begin{array}{l}\text { 이번 연구 이전에 받은 치료 } \\
\text { 법을 조금 더 선호합니다 }\end{array}$ & $\begin{array}{l}\text { 이전에 받은 치료를 약간 } \\
\text { 선호함 }\end{array}$ \\
\hline Slight preference for the treatment I am receiving now & $\begin{array}{l}\text { 실험 참가 후에 받은 치료 } \\
\text { 를 약간 선호함 }\end{array}$ & $\begin{array}{l}\text { 이번 연구의 치료법을 조금 } \\
\text { 더 선호합니다 }\end{array}$ & $\begin{array}{l}\text { 이번 연구의 치료방법을 } \\
\text { 약간 선호함 }\end{array}$ \\
\hline Definitely prefer the treatment I am receiving now & $\begin{array}{l}\text { 실험 참가 후에 받은 치료 } \\
\text { 를 확실하게 선호함 }\end{array}$ & $\begin{array}{l}\text { 분명히 이번 연구의 치료법 } \\
\text { 을 선호합니다 }\end{array}$ & $\begin{array}{l}\text { 이번 연구의 치료방법을 } \\
\text { 분명히 선호함 }\end{array}$ \\
\hline $\begin{array}{l}\text { 9. Would you be willing to use the same treatment for } \\
\text { your bladder problem that you have received since } \\
\text { entering this study? }\end{array}$ & $\begin{array}{l}\text { 9. 귀하의 방광 문제를 치료하 } \\
\text { 기 위해 실험 참가 후에 받 } \\
\text { 으신 거와 동일한 치료를 } \\
\text { 받기를 원하십니까? }\end{array}$ & $\begin{array}{l}\text { 9. 귀하께서는 방광 문제 치료 } 9 \\
\text { 방법으로 이번 연구에서 받 } \\
\text { 으신 치료법과 동일한 치료 } \\
\text { 법을 사용하시겠습니까? }\end{array}$ & $\begin{array}{l}\text { 귀하는 방광 문제를 치료 } \\
\text { 하기 위해 이번 연구에서 } \\
\text { 받은 치료방법과 동일한 } \\
\text { 치료를 받을 의향이 있으 } \\
\text { 십니까? }\end{array}$ \\
\hline Definitely would not use the same treatment again & $\begin{array}{l}\text { 확실히 같은 치료를 받고 } \\
\text { 싶지 않음 }\end{array}$ & $\begin{array}{l}\text { 절대로 동일한 치료법을 사 } \\
\text { 용하지 않을 것이다 }\end{array}$ & $\begin{array}{l}\text { 동일한 치료방법을 분명 } \\
\text { 히 받지 않을 것임 }\end{array}$ \\
\hline Might not use the same treatment again & $\begin{array}{l}\text { 같은 치료를 다시 선택하지 } \\
\text { 않을 수도 있음 }\end{array}$ & $\begin{array}{l}\text { 아마도 동일한 치료법을 사 } \\
\text { 용하지 않을 것이다 }\end{array}$ & $\begin{array}{l}\text { 동일한 치료방법을 아마 } \\
\text { 도 받지 않을 것임 }\end{array}$ \\
\hline
\end{tabular}

(Continued to the next page) 
Table 2. Continued

\begin{tabular}{|c|c|c|c|}
\hline US English item & $\begin{array}{c}\text { First forward Korean } \\
\text { translation (Translation 1) }\end{array}$ & $\begin{array}{l}\text { Second forward Korean } \\
\text { translation (Translation 2) }\end{array}$ & $\begin{array}{l}\text { Reconciled forward } \\
\text { Korean translation }\end{array}$ \\
\hline Might use the same treatment again & $\begin{array}{l}\text { 같은 치료를 다시 선택할 } \\
\text { 수도 있음 }\end{array}$ & $\begin{array}{l}\text { 아마도 동일한 치료법을 사 } \\
\text { 용할 것이다 }\end{array}$ & $\begin{array}{l}\text { 동일한 치료방법을 받을 } \\
\text { 수도 있음 }\end{array}$ \\
\hline Definitely would use the same treatment again & $\begin{array}{l}\text { 확실히 같은 치료를 다시 } \\
\text { 받고 싶음 }\end{array}$ & $\begin{array}{l}\text { 분명히 동일한 치료법을 사 } \\
\text { 용할 것이다 }\end{array}$ & $\begin{array}{l}\text { 동일한 치료방법을 분명 } \\
\text { 히 받을 것임 }\end{array}$ \\
\hline $\begin{array}{l}\text { 10. Would you recommend this treatment to others } \\
\text { with overactive bladder? }\end{array}$ & $\begin{array}{l}\text { 10. 귀하가 받고 계신 치료를 } \\
\text { 다른 과민성방광 환자들에 } \\
\text { 게 추천하시겠습니까? }\end{array}$ & $\begin{array}{l}\text { 10. 귀하께서는 다른 분에게 } \\
\text { 과민성방광 치료방법으로 } \\
\text { 이 치료법을 추천하시겠습 } \\
\text { 니까? }\end{array}$ & $\begin{array}{l}\text { 10. 귀하는 다른 분에게 과 } \\
\text { 민성방광 치료방법으로 } \\
\text { 이 치료방법을 추천하 } \\
\text { 시겠습니까? }\end{array}$ \\
\hline Definitely would not recommend & 추천하지 않을 것이 확실함 & 절대로 추천하지 않을 것이다 & 절대로 추천하지 않을 것임 \\
\hline Might not recommend & 추천하지 않을 수도 있음 & 아마도 추천하지 않을 것이다 & 아마도 추천하지 않을 것임 \\
\hline Might recommend & 추천할 수도 있음 & 아마도 추천할 것이다 & 추천할 수도 있음 \\
\hline Definitely would recommend & 추천할 것이 확실함 & 분명히 추천할 것이다 & 분명히 추천할 것임 \\
\hline $\begin{array}{l}\text { 11. Overall, how satisfied or dissatisfied are you with } \\
\text { this treatment? }\end{array}$ & $\begin{array}{l}\text { 11. 전반적으로 귀하는 귀하의 } \\
\text { 치료에 얼마나 만족 혹은 } \\
\text { 불만족하십니까? }\end{array}$ & $\begin{array}{l}\text { 11. 전체적으로 이 치료법에 } \\
\text { 대해 어느 정도 만족 혹은 } \\
\text { 불만족하십니까? }\end{array}$ & $\begin{array}{l}\text { 11. 전반적으로 귀하의 치 } \\
\text { 료에 얼마나 만족 혹은 } \\
\text { 불만족하십니까? }\end{array}$ \\
\hline Extremely dissatisfied & 완전 불만족 & 아주 매우 불만족스럽다 & 지극히 불만족 \\
\hline Very dissatisfied & 매우 불만족 & 아주 불만족스럽다 & 매우 불만족 \\
\hline Dissatisfied & 불만족 & 불만족스럽다 & 불만족 \\
\hline Satisfied & 만족 & 만족스럽다 & 만족 \\
\hline Very satisfied & 매우 만족 & 아주 만족스럽다 & 매우 만족 \\
\hline Extremely satisfied & 완전 만족 & 아주 매우 만족스럽다 & 지극히 만족 \\
\hline
\end{tabular}

some are the side effects of the treatment you take to treat your overactive bladder?" in the original version, was translated as "gwihaneun gwaminseong banggwang chiryoreul banneundeisseoseo chiryobujagyongi eolmana bulpyeonhasimnikka?" The responses included phrases such as "extremely bothersome," "very bothersome," "somewhat bothersome," "a little bothersome," and "not at all bothersome", which were translated as follows after the panel discussed the translation possibilities: "jigeuki bulpyeonham," "maeu bulpyeonham," "daso bulpyeonham," "yakgan bulpyeonham," and "jeonhyeo bulpyeonhaji aneum," respectively. Question 7, "To what degree have side effects affected your overall satisfaction with treatment?" in the original version, was directly translated as "jeonbanjeogin chiryo manjokdoe bujagyongi eolmana yeonghyangeul michyeotseumnikka?" The translation of this question did not meaningfully differ between the 2 translators. Question 8, "Do you prefer the treatment that you received since entering this study to the treatment you received before the study?" in the original version, was directly translated as "ibeon yeongueseobadeun chiryobangbeobeul deo seonhohasimnikka?" Question 9, "Would you be willing to use the same treatment for your bladder problem that you have re- ceived since entering this study?" in the original version, was directly translated as "dongilhan chiryoreul badeul uihyangi isseusimnikka?" ("Would you be willing to use the same treatment?') The panel discussed and reconciled the 2 different translated sentences into the final question, and included " $u i h y$ ang" ('willing'). Question 10, "Would you recommend this treatment to others with overactive bladder?" in the original version, was directly translated as "ichiryobangbeobeul chucheon hasigetseumnikka?" ("Would you recommend this treatment to others?'). Question 11, “Overall, how satisfied or dissatisfied are you with this treatment?" in the original version, was directly translated as "jeonbanjeogeuro gwihaui chiryoeeol manamanjok hogeun bulmanjokasimnikka?" The panel attempted to choose sentences that preserved the exact meaning of the English phrases, and reconciled the 2 translated versions to provide more natural expressions in the Korean language, within the framework of conceptual equivalence. The panel chose the final forward-translated versions on this basis.

\section{Backward Translation}

As shown in Table 3, the panel agreed that there were no signif- 
Table 3. Backward translation

\begin{tabular}{llc}
\hline Original & \multicolumn{1}{c}{ Forward translation } & Back translation \\
\hline TS-VAS & 치료 만족도 시각적 척도 \\
$\begin{array}{l}\text { Please place a vertical mark on the line to } \\
\text { indicate your answer to the question below }\end{array}$ & $\begin{array}{c}\text { 다음 질문을 답하기 위해 선 위에 세로선을 표기 } \\
\text { 해주시기 바랍니다. }\end{array}$ & $\begin{array}{c}\text { In order to answer the following question, please } \\
\text { indicate a vertical line on the line }\end{array}$ \\
$\begin{array}{ll}\text { Are you satisfied with your treatment? } \\
\text { No, not at all }\end{array}$ & 귀하는 치료에 만족하십니까? & Are you satisfied with your treatment? \\
Yes, completely & 아니요, 전혀 그렇지 않음 & No, I do not feel satisfied at all. \\
OAB-SAT-q & 네, 전적으로 만족함 & Yes, I am fully satisfied. \\
Instructions: & 과민성방광 치료 만족도 설문지 & The satisfaction of overactive bladder treatment \\
& 지시사항: & Explanation/Description:
\end{tabular}

Please think about how satisfied you have been 귀하는 이번 연구에서 받은 치료에 어느 정도 만 Please think how satisfied you are with treatwith the treatment you have received in the 족하는지 생각해 보시기 바랍니다. study. ment you have received during this investigation

The questions below will ask you to rate how sat- 아래의 질문들은 귀하가 과민성방광 치료에 어느 정 The questions below will evaluate either how isfied or dissatisfied you are with your treat- 도 만족 또는 불만족 하는지를 평가할 것입니다. ment for overactive bladder. satisfied or dissatisfied you were with the overactive bladder treatment

Thinking about the past four weeks, check one 지난 4주 동안, 귀하의 치료 경험을 가장 정확하 Please indicate the box that best describes your box for each item that best reflects your treat- 게 반영하는 칸을 항목별로 표시하여 주십시오. treatment experience during the past 4 weeks in ment experience. each category

1. How satisfied or dissatisfied are you with the 1. 치료가 귀하의 증상을 완화시키는데 있어서 어 1. How satisfied or dissatisfied are you with way the treatment relieves your symptoms? 느 정도 만족 혹은 불만족하십니까? treatment relieving your symptoms?

2. How satisfied or dissatisfied are you with the 2. 치료 효과가 나타나는 데까지 걸리는 시간에 2. How satisfied or dissatisfied are you with the amount of time it takes the treatment to start 어느 정도 만족 혹은 불만족하십니까? time that it takes to show the effect of the working? treatment

3. How satisfied or dissatisfied are you with the 3. 귀하가 기대했던 바에 비하여 치료 효과에 어 3. Compared to your expectations, how satisfied effectiveness of the treatment, compared to 느 정도 만족 혹은 불만족하십니까? what you expected?

Extremely dissatisfied

지극히 불만족

Very dissatisfied

Dissatisfied

Satisfied

Very satisfied

Extremely satisfied

\section{매우 불만족}

불만족

만족

매우 만족

지극히 만족 or dissatisfied are you with the effect of the treatment?

Extremely dissatisfied

Very dissatisfied

Dissatisfied

Satisfied

Very satisfied

Fully Satisfied

4. How convenient or inconvenient is it to take 4. 귀하가 치료를 받는데 어느 정도 편리 혹은 불 the treatment as instructed? 편하셨습니까?

Extremely inconvenient

Very inconvenient

Inconvenient

Convenient

Very convenient

Extremely convenient
지극히 불편함

매우 불편함

불편함

편리함

매우 편리함

지극히 편리함
4. How comfortable or uncomfortable are you with receiving treatments the way you have been explained

Extremely uncomfortable

Very uncomfortable

Uncomfortable

Convenient

Very convenient

Exceptionally convenient

5. How often did you experience side effects from 5. 귀하는 얼마나 자주 치료 부작용을 경험하셨습 5. How often have you experienced the side efyour treatment? 니까?

전혀 없음

거의 없음 fects (of the treatment)?

Never

Seldom/rarely

A little of the time

(Continued to the next page) 
Table 3. Continued

\begin{tabular}{|c|c|c|}
\hline Original & Forward translation & Back translation \\
\hline Some of the time & 가끔 있음 & Occasionally \\
\hline A good bit of time & 상당히 있음 & Quite often \\
\hline Most of time & 매우 자주 있음 & Very often \\
\hline All of the time & 항상 있음 & All of the time \\
\hline \multicolumn{3}{|c|}{$\begin{array}{l}\text { 6. How bothersome are the side effects of the } 6 \text {. 귀하는 과민성방광 치료를 받는데 있어서 (치 } 6 \text {. How uncomfortable are you with the side ef- } \\
\text { treatment you take to treat your overactive } \\
\begin{array}{ll}\text { 료) 부작용이 얼마나 불편하십니까? } & \text { fects from/when receiving the overactive } \\
\text { bladder? } & \text { bladder treatment }\end{array}\end{array}$} \\
\hline \multicolumn{3}{|c|}{$\begin{array}{l}\text { 7. To what degree have side effects affected your } 7 \text {. 귀하의 전반적인 치료 만족도에 부작용이 얼마 7. To what extent did the side effects influence } \\
\begin{array}{ll}\text { overall satisfaction with treatment? } & \text { 나 영향을 미쳤습니까? }\end{array}\end{array}$} \\
\hline A great deal & 상당히 많이 & To a very large extent \\
\hline Quite a bit & 꽤 많이 & To a large extent \\
\hline Somewhat & 다소 & To some extent \\
\hline Minimally & 극히 일부분 & To a very small extent \\
\hline Not at all & 전혀 영향 없음 & No influence at all \\
\hline \multicolumn{3}{|c|}{$\begin{array}{l}\text { 8. Do you prefer the treatment that you received 8. 귀하는 이전에 받았던 치료방법보다 이번 연구 8. Do you prefer this treatment (method) rather } \\
\text { since entering this study to the treatment you 에서 받은 치료방법을 더 선호하십니까? } \\
\text { received before the study? }\end{array}$} \\
\hline $\begin{array}{l}\text { 9. Would you be willing to use the same treat- } 9 \\
\text { ment for your bladder problem that you have } \\
\text { received since entering this study? }\end{array}$ & $\begin{array}{l}\text { 9. 귀하는 방광 문제를 치료하기 위해 이번 연구 } \\
\text { 에서 받은 치료방법과 동일한 치료를 받을 의 } \\
\text { 향이 있으십니까? }\end{array}$ & $\begin{array}{l}\text { 9. Do you have any intentions to receive the } \\
\text { same type/method of treatment as the previ- } \\
\text { ous one to treat the bladder problems? }\end{array}$ \\
\hline $\begin{array}{l}\text { 10. Would you recommend this treatment to } 1 \\
\text { others with overactive bladder? }\end{array}$ & $\begin{array}{l}\text { 10. 귀하는 다른 분에게 과민성방광 치료방법으 } 1 \\
\text { 로 이 치료방법을 추천하시겠습니까? }\end{array}$ & $\begin{array}{l}\text { 10. Would you recommend this overactive blad- } \\
\text { der treatment (method) to other people? }\end{array}$ \\
\hline $\begin{array}{l}\text { 11. Overall, how satisfied or dissatisfied are you } 1 \\
\text { with this treatment? }\end{array}$ & $\begin{array}{l}\text { 11. 전반적으로 귀하의 치료에 얼마나 만족 혹은 } 1 \\
\text { 불만족하십니까? }\end{array}$ & $\begin{array}{l}\text { 11. How satisfied or dissatisfied are you with } \\
\text { your treatment overall? }\end{array}$ \\
\hline
\end{tabular}

TS-VAS, Treatment Satisfaction Visual Analogue Scale; OAB-SAT-q, Overactive Bladder Satisfaction with Treatment Questionnaire.

icant differences between the wording of the sentences in the original and the back-translated versions.

\section{Cognitive Debriefing and Proofreading}

The translation was tested by 5 patients with $\mathrm{OAB}$ ( 3 men and 2 women; age range, 30-70 years) who underwent medical therapy. Their levels of education varied from being a high school graduate to a university graduate. All patients completed the questionnaires within 8 minutes. One patient reported being unsure about the marking position on the line of TS-VAS. He suggested that vertical lines on the long horizontal line of TSVAS could help patients understand the scale and mark their responses. The panel accepted his proposal and added 10 vertical lines indicating 0 to 10 points. Three patients reported that the translated questions were clear and easy to understand; however, 2 patients felt that the questions were a bit difficult to understand owing to the excessively long structure of the ques-

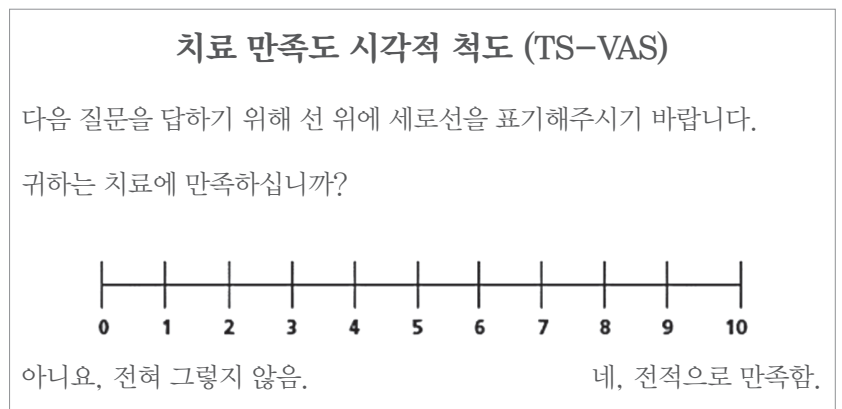

Fig. 1. Korean version of the Treatment Satisfaction Visual Analogue Scale (TS-VAS).

tions. The panel discussed these opinions that were articulated during the cognitive debriefing process, and the final versions of the questionnaires are shown in Figs. 1 and 2. 


\section{과민성방광 치료만족도 설문지}

지시사항: 귀하는 이번 연구에서 받은 치료에 어느 정도 만족하는지 생각해 보시기 바랍니다. 아래의 질문들은 귀하가 과민성 방광 치료에 어느 정도 만 족 또는 불만족 하는지를 평가할 것입니다. 지난 4주 동안, 귀하의 치료 경험을 가장 정확하게 반영하는 칸을 항목별로 표시하여 주십시오.

\begin{tabular}{|c|c|c|c|c|c|c|}
\hline & 지극히 불만족 & 매우 불만족 & 불만족 & 만족 & 매우 만족 & 지극히 만족 \\
\hline \multirow{2}{*}{$\begin{array}{l}\text { 1. 치료가 귀하의 증상을 완화시키는데 있어 } \\
\text { 서 어느 정도 만족 혹은 불만족하십니까? }\end{array}$} & 口 & 口 & 口 & 口 & $\square$ & $\square$ \\
\hline & 1 & 2 & 3 & 4 & 5 & 6 \\
\hline \multirow{2}{*}{$\begin{array}{l}\text { 2. 치료 효과가 나타나는 데까지 걸리는 시간 } \\
\text { 에 어느 정도 만족 혹은 불만족하십니까? }\end{array}$} & 口 & 口 & 口 & 口 & 口 & 口 \\
\hline & 1 & 2 & 3 & 4 & 5 & 6 \\
\hline \multirow{2}{*}{$\begin{array}{l}\text { 3. 귀하가 기대했던 바에 비하여 치료 효과에 } \\
\text { 어느 정도 만족 혹은 불만족하십니까? }\end{array}$} & 口 & 口 & 口 & 口 & 口 & 口 \\
\hline & 1 & 2 & 3 & 4 & 5 & 6 \\
\hline
\end{tabular}

4. 귀하가 치료를 받는데 어느 정도 편리 혹 지극히 불편함 은 불편하셨습니까?

5. 귀하는 얼마나 자주 치료 부작용을 경험하 셨습니까?

6. 귀하는 과민성방광 치료를 받는데 있어서 (치료) 부작용이 얼마나 불편하십니까?

7. 귀하의 전반적인 치료 만족도에 부작용이 얼마나 영향을 미쳤습니까?

8. 귀하는 이전에 받았던 치료방법보다 이번 연구에서 받은 치료방법을 더 선호하십니 까?

9. 귀하는 방광 문제를 치료하기 위해 이번 연구에서 받은 치료방법과 동일한 치료를 받을 의향이 있으십니까?

10. 귀하는 다른 분에게 과민성방광 치료방 법으로 이 치료방법을 추천하시겠습니 까?

$\square$

1

전혀 없음

거의 없음

口

$\square$

0

매우 불편함

불편함

편리함

매우 편리함

지극히 편리함

'전혀없음' 이면

8번 문항으로

가세요

지극

$\square$

3

가끔 있음

$\square$

4

$\square$

5

$\square$

6

상당히 있음 매우 자주 있음

항상 있음

1

$\square$
2

$\square$

3

\section{$\square$}

4

$\square$

5

자

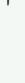

ㅁ

$\square$
1

과민성방광 치료 이전에 받은 치 이전에 받은 치 이번 연구의 치 이번 연구의 치 를 받아본 적이 료를 분명히 선 료를 약간 선호 료방법을 약간 료방법을 분명히

$\begin{array}{ccccc}\text { 없음 } & \text { 효함 } & \text { 함 } & \text { 선호함 } & \text { 선호함 } \\ \square & \square & \square & \square & \square\end{array}$

0

1

2

3

4

동일한 치료방법 동일한 치료방법 동일한 치료방법 동일한 치료방법 을 분명히 받지 을 아마도 받지 을 받을 수도 을 분명히 받을 않을 것임 않을 것임 있음 것임

$\begin{array}{cccc}\square & \square & \square & \square \\ 1 & 2 & 3 & 4 \\ \begin{array}{c}\text { 절대로 추천하지 } \\ \text { 않을 것임 }\end{array} & \text { 않음 출천핫임 } & \text { 추천할 수도 } & \text { 분명히 추천할 } \\ \text { 있음 } & \text { 것임 }\end{array}$

$\begin{array}{llll}\square & \square & \square & \square \\ 1 & 2 & 3 & 4\end{array}$

11. 전반적으로 귀하의 치료에 얼마나 만족 지극히 불만족 혹은 불만족하십니까?
불만족

만족 


\section{DISCUSSION}

This study presented the first linguistic validation of the TSVAS and OAB-SAT-q, which are used to evaluate patients' satisfaction with their treatment.

Linguistic validation is the process of investigating the reliability, conceptual equivalence, and content validity of translations of PRO measures. According to the universal translation methodology, the process of validation consists of permission for translation, forward translation, reconciliation, back-translation, cognitive debriefing, and proofreading [11]. Forward translations were performed simultaneously by 2 separate bilingual speakers. One was a 19-year-old Korean woman who had lived in Phuket, Thailand for 17 years. The other was a 24-yearold Korean man who had lived in the state of Maryland in the USA for 4.5 years, before becoming a university student majoring in Business Administration at Korea University. The forward translators in the present study were experienced bilingual speakers who were involved in the forward translation process for other questionnaires. Subsequently, reconciliation was performed to resolve discrepancies between the 2 forward translations. The reconciler altered the forward translations to make them suitable, or offered new forward translations, if necessary. The back-translation of the reconciled version involved another bilingual speaker who was fluent in the Korean and English languages, but was not involved in the previous steps of the forward translation process. Cognitive debriefing interviews were performed to gather data on the patients' interpretation of the translation. This process ensured that the meaning intended by the developer was retained in translation and was also understood by the patients in the same way [11]. The authors performed the validation process according to this universal process of translation validation.

The VAS is a valid and reliable measure for rating pain, and is widely accepted as a way to quantitatively evaluate QoL [12]. In adults, it has been observed to be a responsive and valid tool for measuring satisfaction, feelings, and emotional function [8,13-15]. VAS-based questionnaires could be a useful tool for assessing satisfaction specific to treatment with any medication [8]. The TS-VAS, which is widely used to evaluate patient satisfaction, reflects a patient's subjective perceptions or opinions regarding the treatment experience [8].

$\mathrm{OAB}$ is a condition that negatively effects patients' well-being, and is a chronic disease that requires long-term medical therapy. Patient satisfaction is an important issue when deter- mining whether to continue a drug for the treatment of OAB. Therefore, it is important for physicians to evaluate patient satisfaction.

Previous investigators have used King's Health Questionnaire [16], the OAB questionnaire [17], the Overactive Bladder Symptoms Score [18] and Benefit, Satisfaction, and Willingness to Continue questionnaires to evaluate the severity of the symptoms of $\mathrm{OAB}$, their effect on QoL, and patients' satisfaction with their treatment [19]. The OAB-SAT-q is a useful assessment tool for evaluating satisfaction among patients receiving treatment for OAB [10]. The OAB-SAT-q was developed by identifying concepts that are important for treatment satisfaction among patients with $\mathrm{OAB}$. The questionnaire was developed and refined based on a review of existing instruments measuring treatment satisfaction, input from physicians experienced in treating $\mathrm{OAB}$, and patient feedback [10]. Therefore, the findings of the current study will be helpful to domestic investigators, who will use the Korean version of the TS-VAS and OAB-SAT-q to assess treatment satisfaction and response among patients.

A limitation of this study is that it did not include a reliability analysis, which would enhance the strength of the association between the scores obtained from the English- and Koreanlanguage versions. However, the content of the original questionnaires was not modified, and a reliability test can be considered for psychometric validation in the future.

In conclusion, the present study reports the successful linguistic validation of the TS-VAS and OAB-SAT-q. Our results can help provide reliable PROs to physicians in Korea.

\section{REFERENCES}

1. Golin CE, DiMatteo MR, Gelberg L. The role of patient participation in the doctor visit. Implications for adherence to diabetes care. Diabetes Care 1996;19:1153-64.

2. Katz JN. Patient preferences and health disparities. JAMA 2001; 286:1506-9.

3. Owens DK. Spine update. Patient preferences and the development of practice guidelines. Spine (Phila Pa 1976) 1998;23:1073-9.

4. Stach-Lempinen B, Kujansuu E, Laippala P, Metsänoja R. Visual analogue scale, urinary incontinence severity score and $15 \mathrm{D}$--psychometric testing of three different health-related quality-of-life instruments for urinary incontinent women. Scand J Urol Nephrol 2001;35:476-83.

5. Ohnhaus EE, Adler R. Methodological problems in the measure- 
ment of pain: a comparison between the verbal rating scale and the visual analogue scale. Pain 1975;1:379-84.

6. Abdel-Fattah M, Ramsay I, Barrington JW. A simple visual analogue scale to assess the quality of life in women with urinary incontinence. Eur J Obstet Gynecol Reprod Biol 2007;133:86-9.

7. Hayes MH, Patterson DG. Experimental development of the graphic rating method. Psychol Bull 1921;18:98-9.

8. Chapman CR, Casey KL, Dubner R, Foley KM, Gracely RH, Reading AE. Pain measurement: an overview. Pain 1985;22:1-31.

9. Brokelman RB, Haverkamp D, van Loon C, Hol A, van Kampen A, Veth $R$. The validation of the visual analogue scale for patient satisfaction after total hip arthroplasty. Eur Orthop Traumatol 2012;3: 101-5.

10. Margolis MK, Fox KM, Cerulli A, Ariely R, Kahler KH, Coyne KS. Psychometric validation of the overactive bladder satisfaction with treatment questionnaire (OAB-SAT-q). Neurourol Urodyn 2009; 28:416-22.

11. Eremenco SL, Cella D, Arnold BJ. A comprehensive method for the translation and cross-cultural validation of health status questionnaires. Eval Health Prof 2005;28:212-32.

12. Stinson JN, Kavanagh T, Yamada J, Gill N, Stevens B. Systematic review of the psychometric properties, interpretability and feasibility of self-report pain intensity measures for use in clinical trials in children and adolescents. Pain 2006;125:143-57.
13. Guyatt GH, Townsend M, Berman LB, Keller JL. A comparison of Likert and visual analogue scales for measuring change in function. J Chronic Dis 1987;40:1129-33.

14. Jaeschke R, Singer J, Guyatt GH. A comparison of seven-point and visual analogue scales. Data from a randomized trial. Control Clin Trials 1990;11:43-51.

15. Singer AJ, Thode HC Jr. Determination of the minimal clinically significant difference on a patient visual analog satisfaction scale. Acad Emerg Med 1998;5:1007-11.

16. Margolis MK, Vats V, Coyne KS, Kelleher C. Establishing the content validity of the King's Health Questionnaire in men and women with overactive bladder in the US. Patient 2011;4:177-87.

17. Oh SJ, Son H, Kim SW, Lee KS, Choo MS, Kim SO, et al. Psychometric properties of the Korean Version of the Overactive Bladder Questionnaire (OAB-q) in a Korean population. Int Neurourol J 2012;16:77-85.

18. Homma Y, Yoshida M, Seki N, Yokoyama O, Kakizaki H, Gotoh M, et al. Symptom assessment tool for overactive bladder syndrome: overactive bladder symptom score. Urology 2006;68:318-23.

19. Pleil AM, Coyne KS, Reese PR, Jumadilova Z, Rovner ES, Kelleher CJ. The validation of patient-rated global assessments of treatment benefit, satisfaction, and willingness to continue--the BSW. Value Health 2005;8 Suppl 1:S25-34. 